\section{Smoggefahr: Wer ist gefährdet?}

Belastung schließen. Vermutlich handelt es sich aber nicht um einen reinen $\mathrm{SO}_{2}$-Effekt - vielmehr scheint auch die mit einer erhöhten $\mathrm{SO}_{2}$-Konzentration einhergehende Partikelbelastung mitverantwortlich sein.

\section{Partikel}

Partikelförmige Luftverunreinigungen entstehen u.a. aus Feuerungsanlagen, Industrie- oder Autoabgasen, aber auch durch Kondensation oder Sublimation gasförmiger Stoffe. Ihre Relevanz wurde lange Zeit unterschätzt. Erst jetzt laufen intensive Forschungen zur Bedeutung partikelförmiger Luftbestandteile für die Entstehung und Aufrechterhaltung von Krankheiten.

Daten aus klinisch-experimentellen Provokationstestungen mit Partikeln aus der Außenluft sind nur vereinzelt verfügbar - so bestehen beispielsweise Hinweise auf entzündliche Veränderungen in den Atemwegen nach Exposition gegenüber hohen Konzentrationen von Partikeln aus Dieselabgasen. Diese Entzündungsreaktionen führen auch zu Veränderungen der Blutrheologie und werden heute für die unter erhöhter Partikelbelastung beobachtete Zunahme der kardiovaskulären Mortalität verantwortlich gemacht.

In der Epidemiologie sind Kurzzeiteffekte der Schwebstaubbelastung in der Außenluft aus historischen Smogepisoden seit längerem bekannt. Aktuelle Untersuchungen lassen eine Korrelation zwischen der mittleren Konzentration von Schwefelfeinstäuben $\left(\mathrm{PM}_{10}\right.$ particulate matter $<10$ $\mu \mathrm{m})$ einerseits sowie der Gesamtmortalität, der respiratorischen und kardiovaskulären Mortalität, der Häufigkeit von Krankenhauseinweisungen mit respiratorischen Erkrankungen und dem Ausmaß von Lungenfunktionseinschränkungen erkennen. Dies gilt sowohl für Asthmatiker wie auch für die Allgemeinbevölkerung. Dabei sind die Ergebnisse der Einzelstudien erstaunlich kongruent. So wuchs die tägliche Sterblichkeitsrate um 0,5 bis $1,5 \%$, wenn die Feinstaubbelastung um 10 $\mu \mathrm{g} / \mathrm{m}^{3}$ anstieg.

bk stisch gesichert.

\section{Die Daten einiger epidemiologi-} scher Studien stützen diese Beobachtiker bei erhöhten $\mathrm{NO}_{2}$-Konzentrationen in der Außenluft mit zunehmenden Atemwegsbeschwerden reagieren könzwischen einer Konfrontation mit bei uns vorkommenden erhöhten $\mathrm{NO}_{2}-$ Konzentrationen und Parametern der Morbidität als statihauseinweisungen und Sterblichkeit speziell unter längerdauernder $\mathrm{SO}_{2}$ - 